\title{
Configurational Entropy and Diffusivity of Supercooled Water
}

\author{
Antonio Scala ${ }^{1}$, Francis W. Starr ${ }^{1, *}$, Emilia La Nave ${ }^{1}$, \\ Francesco Sciortino ${ }^{2}$ and H. Eugene Stanley ${ }^{1}$ \\ ${ }^{1}$ Center for Polymer Studies, Center for Computational Science, and Department of Physics, \\ Boston University, Boston, MA 02215 USA \\ 2 Dipartimento di Fisica e Istituto Nazionale per la Fisica della Materia, \\ Universitá di Roma La Sapienza, P.le Aldo Moro 2, I-00185, Roma, Italy
}

(September 18, 2018)

We calculate the configurational entropy $S_{\text {conf }}$ for the $\mathrm{SPC} / \mathrm{E}$ model of water for state points covering a large region of the $(T, \rho)$ plane. We find that (i) the $(T, \rho)$ dependence of

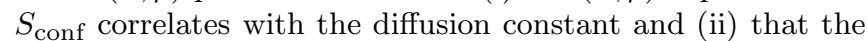
line of maxima in $S_{\text {conf }}$ tracks the line of density maxima. Our simulation data indicate that the dynamics are strongly influenced by $S_{\text {conf }}$ even above the mode-coupling temperature $T_{\mathrm{MCT}}(\rho)$.

PACS numbers: 61.43.Fs, 64.70.Pf, 65.50.+m, 66.20.+d

Computer simulation studies are contributing to the understanding of the slow dynamical processes in simple and molecular liquids on approaching the glass transition [1]. In particular, the space, time and temperature dependence of many dynamical quantities have been calculated and compared with the predictions of the modecoupling theory (MCT) [2], which provides a description of the dynamics in weakly supercooled states [3] of many atomic and molecular liquids, including the SPC/E model of water studied here 4.5 .

Simulations have also been used to investigate the "thermodynamic approach" to the glass transition [6 8], which envisages a relation between diffusion constant $D$ and configurational entropy $S_{\text {conf }}$ by relating the dynamics of liquids at low $T$ to the system's exploration of its configuration space. The properties of the liquid are dominated by the basins of attraction of local potential energy minima; the liquid experiences vibrations localized around a basin and rearranges via relatively infrequent inter-basin jumps [9]. Motivated by the separation of time scales, the total entropy $S$ may be separated into two parts: (i) an intra-basin contribution $S_{\text {vib }}$, which measures the vibrational entropy of a system constrained to reside within a basin, and (ii) a configurational contribution, $S_{\text {conf }}$, quantifying the multiplicity of basins [10]. Explicit calculation of $S_{\text {conf }}$ has been per-

\footnotetext{
* Current Address: Polymers Division and Center for Theoretical and Computational Materials Science, National Institute of Standards and Technology, Gaithersburg, Maryland 20899
}

formed for hard spheres [8], soft spheres [7], LennardJones systems 11, 12 and tetravalent network glasses 13] with the aim of evaluating the Kauzmann temperature $T_{K}$, at which $S_{\text {conf }}$ appears to vanish 14.

One of the most studied models of molecular liquids is the SPC/E potential, designed to mimic the behavior of water [15]. The dynamical properties of this model have been studied in detail in the weakly supercooled regime and have been shown to be consistent with the predictions of MCT [4.5]. The SPC/E potential is of particular interest for testing theories of the supercooled-liquid dynamics, since, as observed experimentally for water, along isotherms $D$ has a maximum as function of the pressure $P$ or of the density $\rho$ [16]. This maximum becomes more pronounced upon cooling. Furthermore, the line of isobaric density maxima, which corresponds to the line of isothermal entropy maxima from the Maxwell relation $(\partial V / \partial T)_{P}=-(\partial S / \partial P)_{T}$, appears strongly correlated to the line of $D$ maxima [Fig. 1 (a)], providing motivation to test possible relationships between $S_{\text {conf }}$ and $D$. A test of such relationship, based on the analysis of experimental data at ambient pressure, was performed by Angell and coworkers in 1976 [17].

Here we calculate the entropies $S, S_{\text {vib }}$ and $S_{\text {conf }}$ for the SPC/E potential for state points covering a large region of the $(T, \rho)$ phase diagram. We then compare

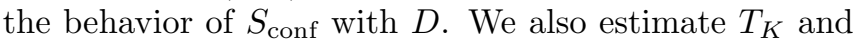
consider its relation with the MCT temperature $T_{\mathrm{MCT}}$. We first calculate $S$ for a reference point $(T=1000 \mathrm{~K}$ and $\left.\rho=1.0 \mathrm{~g} / \mathrm{cm}^{3}\right)[18]$, and then calculate the entropy as a function of $(T, \rho)$ via thermodynamic integration 19 using the state points simulated in Ref. [5], as well as new simulations extending to higher temperatures [20]. We show an example of $S$ along the $\rho=1.0 \mathrm{~g} / \mathrm{cm}^{3}$ isochore in Fig.2(a).

We calculate $S_{\mathrm{vib}}$, i.e. the entropy of the liquid constrained in a typical basin, using the properties of the basins visited in equilibrium by the liquid. For each $(T, \rho)$, we calculate, using the conjugate gradient algorithm [21], the corresponding local minima - called inherent structures (IS) 22] - for 100 configurations [23]. We estimate $S_{\text {vib }}$ by adding anharmonic corrections to the harmonic contribution $S_{\text {harm } 24}$ 


$$
S_{\text {harm }}=k_{B} \sum_{i=1}^{6 N-3}\left[\ln \frac{k_{B} T}{\hbar \omega_{i}}-1\right],
$$

where $\omega_{i}$ are the normal-mode frequencies of the IS determined form the Hessian matrix [25. Ref. [12] found that the harmonic approximation for a binary LennardJones mixture is a valid estimate of $S_{\text {vib }}$ for temperatures around $T_{\mathrm{MCT}}$. However, in the case of the SPC/E potential, we find that there are significant anharmonicities in the basins. This can be seen from the fact that, if the system were purely harmonic, the energy $E$ should equal the energy of a minimum $E_{\text {conf }}$ plus the contribution $E_{\text {harm }}=(6 N-3) k_{B} T / 2$ of the harmonic solid approximation. In contrast with the binary mixture Lennard-Jones case, we find the vibrational contribution $E_{\text {vib }} \equiv E-E_{\text {conf }}$ to be roughly $10 \%$ larger than the harmonic approximation, even at the lowest temperatures studied. We then estimate the anharmonic contributions to $S_{\text {vib }}$ by heating the IS at constant volume and measuring the deviation of $E_{\mathrm{vib}}$ from the harmonic approximation [26]. For each collection of basins corresponding to a particular $(T, \rho)$ state point, we calculate $E_{\mathrm{vib}}$ in the range $T=0-200 \mathrm{~K}$ and fit our results using the approximation

$$
E_{\mathrm{vib}}=E_{\mathrm{harm}}+a T^{2}+b T^{3} .
$$

By integrating the relation $d S_{\mathrm{vib}}=d E_{\mathrm{vib}} / T$, we find

$$
S_{\mathrm{vib}}=S_{\mathrm{harm}}+2 a T+\frac{3}{2} b T^{2}
$$

We use Eq. (3) to extrapolate $S_{\text {vib }}$ to higher temperatures.

¿From the knowledge of $S_{\mathrm{vib}}$, we calculate $S_{\mathrm{conf}}=S-$ $S_{\text {vib. }}$. As an example of the entire procedure, we show in Figs. 2(a) and 2(b) the $T$ dependence of $S_{\text {vib }}$ and $S_{\text {conf }}$ for the $\rho=1.0 \mathrm{~g} / \mathrm{cm}^{3}$ isochore. In Fig. B(a), we show

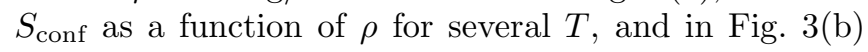
we show the behavior of $D$ along the same isotherms. Fig. 1 shows that at high densities the behavior of $\ln (D)$ is nearly linear when plotted as a function of $\left(T S_{\text {conf }}\right)^{-1}$, as proposed by Adam and Gibbs [6] over 30 years ago.

Fig. 1 (b) shows the lines of maxima for $D$ and $S_{\text {conf }}$ in the region $T \leq 260 \mathrm{~K}$, where we have been able to clearly detect a maximum. To highlight the differences between $S$ and $S_{\text {conf }}$, we show also the line at which $S(\rho, T)$ has a maximum. Fig. 1 1 (b) shows that the lines of maxima in $D$ and in $S_{\text {conf }}$ track each other within the uncertainties of our calculations.

Note that the density where $D$ has a maximum $(\rho \approx$ $\left.1.15 \mathrm{~g} / \mathrm{cm}^{3}\right)$, as well as the density where the maximum

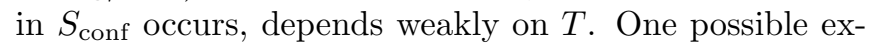
planation is that, for the values of $\rho$ where the maxima in $D$ and $S_{\text {conf occur, two density-dependent (and primarily }}$ $T$ independent) mechanisms balance. Increasing $\rho$ in water from the "ideal" tetrahedral density (i.e., that of ice
Ih, about $0.92 \mathrm{~g} / \mathrm{cm}^{3}$ ) leads to the progressive destruction of the hydrogen-bond network, and hence increases the number of minima (and therefore $\mathrm{S}_{\text {conf }}$ ), since there are more configurations corresponding to a disordered tetrahedral network. However, at large enough density, core repulsion begins to dominate the liquid properties, as expected for "typical" liquids. In such a case, one expects that increasing $\rho$ decreases the number of minima in the potential energy landscape, as the system becomes more densely packed, and hence fewer configurations are possible.

We observe that the close connection between $D$ and $S_{\text {conf }}$ shown in Fig. 3 occurs in the same region where the dynamics of SPC/E water can be rather well described by $\mathrm{MCT}$, suggesting that MCT may be able to capture the reduction of the mobility due to entropic effects. Moreover, in the same region of the $(T, \rho)$ plane, $D$ also correlates well with the number of directions in configuration space connecting different basins [27]. This suggests the possibility of a statistical relation between the number of minima and their connectivity [28].

Before concluding, we note that the present approach allows us to estimate the locus of the Kauzmann temperature $T_{K}(\rho)$ at which $S_{\text {conf }}$ would disappear on extrapolating to lower $T$. Fig. 司 shows the calculated values of $T_{K}(\rho)$ together with the locus of mode coupling temperatures $T_{M C T}(\rho)$. The ratio $T_{M C T} / T_{K}$ has been used as an indication of the fragility of a liquid [29]; we find $T_{M C T} / T_{K} \approx 1.05-1.15$ suggesting that $\mathrm{SPC} / \mathrm{E}$ is an extremely fragile liquid in the region of temperatures near $T_{M C T}$ - as also found in experimental measurements. We note that the values of $T_{K}(\rho)$ depend strongly on the validity of the extrapolation of the potential energy [30] to temperatures lower than the one we can equilibrate with the present computer facilities. Slower changes in $E_{\text {conf }}$ below the lowest simulated state point would produce a much slower decrease for $S_{\text {conf }}$ [31]. This may be quite plausible since it appears that $E_{\text {conf }}$ is approaching the crystal value, which is expected to always be less than the liquid value. A much slower change in $S_{\text {conf }}$ is expected to be accompanied by a slower decrease of $D$, which may be related to a possible fragile-to-strong transition in water 32.

We thank C.A. Angell, S. Sastry, and R.J. Speedy for helpful discussions, and the NSF for support; FS acknowledges partial support from MURST (PRIN 98).

[1] See for example K. Binder et al, in Complex Behavior of Glassy Systems, M. Rubí and C. Perez-Vicente Eds. (Springer Berlin 1997); W. Kob, J. Phys. Cond. Matter 11, R85 (1999). 
[2] W. Götze and L. Sjögren, Rep. Prog. Phys. 55, 241 (1992).

[3] We define "weakly supercooled states" as the temperature range where the diffusion constant decreases by 3 or 4 orders of magnitude compared to its normal liquid value $\left(\sim 10^{-5} \mathrm{~cm}^{2} / \mathrm{s}\right)$. When $T$ approaches the dynamical critical temperature predicted by the ideal MCT, the description of the slow dynamical processes offered by the ideal $M C T$ breaks down.

[4] L. Fabbian, et al, Phys. Rev. E xx, xx (1999) and references therein.

[5] F.W. Starr, et al., Phys. Rev. Lett. 82, 3629 (1999).

[6] R.O. Davies and G.O. Jones, Adv. in Physics, 2, 370 (1953).; G. Adams and J.H. Gibbs, J. Chem. Phys. 43, 139 (1958); J.H. Gibbs and E. A. Di Marzio, J. Chem. Phys., 28, 373 (1958).; R.J.Speedy, J. Chem. Phys. 110, 4559 (1999); J. Chem. Phys. B, 103 , 4060 (1999). M. Shultz, Phys. Rev. B 57, 11319 (1998); D.C. Wallace, Phys.Rev. E, 56, 4179 (1997).

[7] M. Mézard and G. Parisi, J.Phys. Cond. Matter 11, A157 (1999) and references therein.

[8] R.J. Speedy, J. Phys. Cond. Matter 10, 4185 (1998); ibid. 9, 8591 (1997); ibid. 8, 10907 (1996).

[9] M. Goldstein, J. Chem. Phys. 51, 3728 (1969).

[10] In the context of spin glasses, the configurational entropy

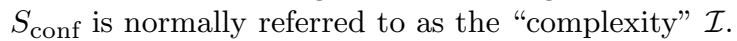

[11] B. Coluzzi, G. Parisi and P. Verrochio, condmat/9904124

[12] F. Sciortino, W. Kob, and P. Tartaglia, Phys. Rev. Lett. xx xx (1999).

[13] R.J. Speedy and P.G. Debenedetti, Mol. Phys. 88, 1293 (1996).

[14] A.W. Kauzmann, Chem. Rev. 43, 219 (1948).

[15] H.J.C. Berendsen, J.R. Grigera, and T.P. Stroatsma, J. Phys. Chem. 91, 6269 (1987).

[16] F.X. Prielmeier, E.W. Lang, R.J. Speedy, and H.-D. L üdemann, Phys. Rev. Lett. 59, 1128 (1987); Ber. Bunsenges. Phys. Chem. 92, 1111 (1988).

[17] C.A. Angell, E.D. Finch, L.A. Woolf and P. Back, J. Chem. Phys. 65, 3063 (1976).

[18] At high $T$ and infinite volume, $S$ is given by the expression for an ideal gas of rigid triatomic molecules. We determine $S$ versus $\rho$ via thermodynamic integration along the $T=1000 K$ isotherm starting from the known analytic value in the infinite volume limit.

[19] Specifically, we integrate the relationship $d S=d E / T+$ $P d V / T$ along both constant $T$ and constant $V$ paths. Furthermore, we have tested the reliability of our results for $S$ by checking that $\oint d S=0$ along several closed $P-V$ cycles.

[20] All the relevant simulation details, such as integration step, potential truncation, and temperature control may be found in ref. [5].

[21] W.H. Press, B.P. Flannery, A.A. Teukolsky and W.T. Vetterling, Numerical Recipes - The Art of Scientific Computing (Cambridge University, Cambridge) (1986).

[22] F.H. Stillinger and T.A. Weber, Phys. Rev. A 25, 978 (1982); F.H. Stillinger and T.A. Weber, Science 225, 983 (1984). F.H. Stillinger, Science, 267 1935, (1995).

[23] The configurations we use span a time of at least 25 times the $\alpha$-relaxation time of the density-density correlation function, for two independent systems.

[24] An evaluation of the harmonic free energy of the inherent structures in simulated water is in A. Pohorille, L.R. Pratt, R. A. LaViolette, M.A. Wilson and R.D. MacElroy, J. Chem. Phys., 87, 6070 (1987).

[25] L.D. Landau and E.M. Lifshits, Statistical Physics (Addison-Wesley, Reading, 1969).

[26] We have checked that systems remain confined in the same basin up to $T \approx 200 K$ when heating the IS from $T=0 \mathrm{~K}$ in the time scale of $200 \mathrm{ps}$. We have evaluated this anharmonic contribution from the IS of the simulations at $T=210 \mathrm{~K}$. However, using the IS generated from higher temperature configurations does not significantly affect our results.

[27] E. La Nave, A. Scala, F.W. Starr, F. Sciortino, and H.E. Stanley, cond-mat/9908412.

[28] L. Angelani, G. Parisi,G. Ruocco and G. Viliani, Phys. Rev. Lett. 81, 4648 (1998).

[29] C.A. Angell, Science 267, 1924 (1995); C.A. Angell, B.E. Richards and V. Velikov, J. Phys. Cond. Mat. 11, A75 (1999) and references therein.

[30] It is convenient to find a functional form to fit the data for $S(T)$. Recently it has been suggested theoretically [Y. Rosenfeld and P. Tarazona, Mol. Phys. 95, 141 (1998)] that — in classical fluids — the $T$ dependence of the potential energy at constant volume follows a $T^{3 / 5}$ law on cooling. This $T$-dependence has been verified in several atomic systems, such as Lennard-Jones, soft spheres, and charged systems in the limit of large densities or low $T$. We find that this $T$ dependence is numerically obeyed also in our molecular system for $200<T<300$.

[31] In the context of estimating $T_{K}$ from the analysis of experimental or numerical data, no distinction can be made between a scenario where $S_{\text {conf }}$ indeed goes to zero at $T_{K}$ (with a clear break in the first $T$ derivative) and a scenario where the extrapolated $S_{\text {conf }}$ has a dramatic change in slope and approaches zero only at a much lower $T$.

[32] C.A. Angell, J. Phys. Chem. 97, 6339 (1993); K. Ito, C.T. Moynihan, and C.A. Angell, Nature 398, 492 (1999).

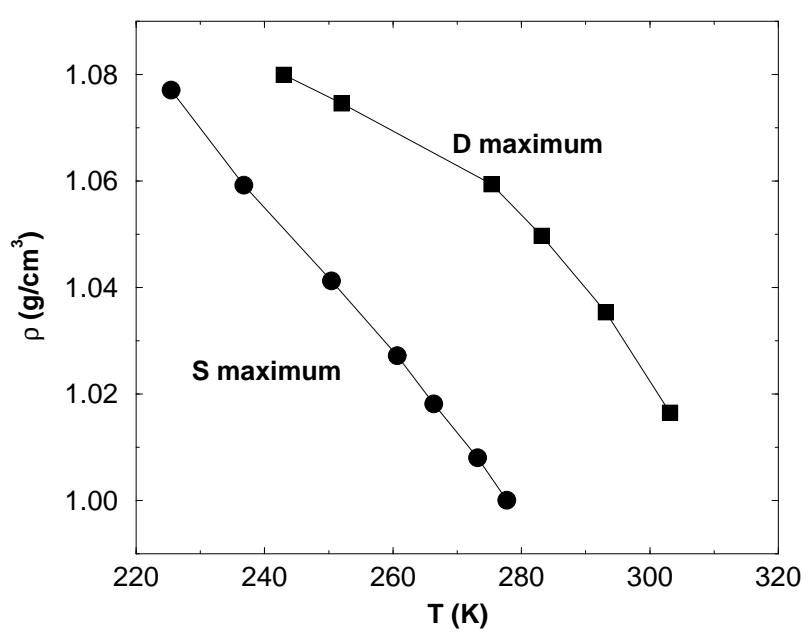




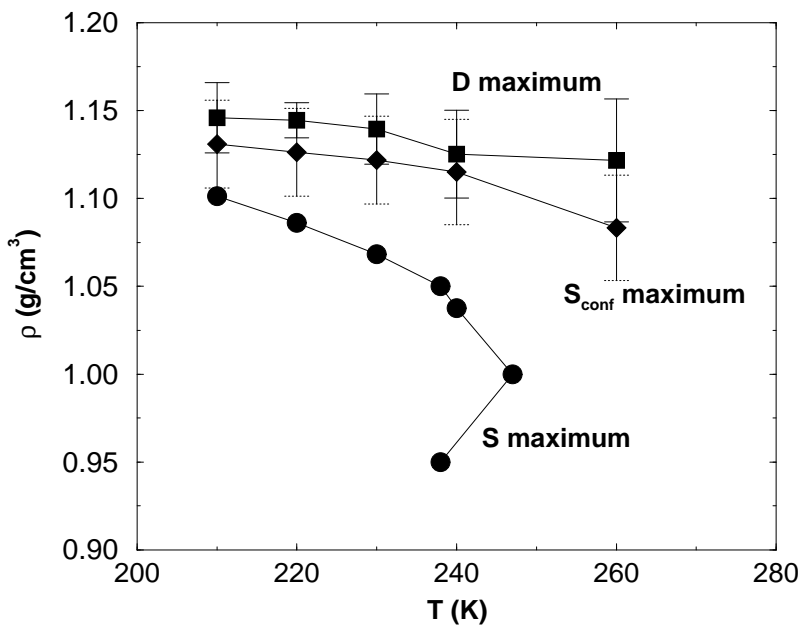

FIG. 1. (a) Line of maxima of the entropy $S$ and of the diffusion constant $D$ from experimental data on water 16 . (b) Lines of entropy maxima $S$, configurational entropy maxima $S_{\text {conf }}$ and diffusion constant maxima $D$ (from Ref. [5]) for the $\mathrm{SPC} / \mathrm{E}$ model.

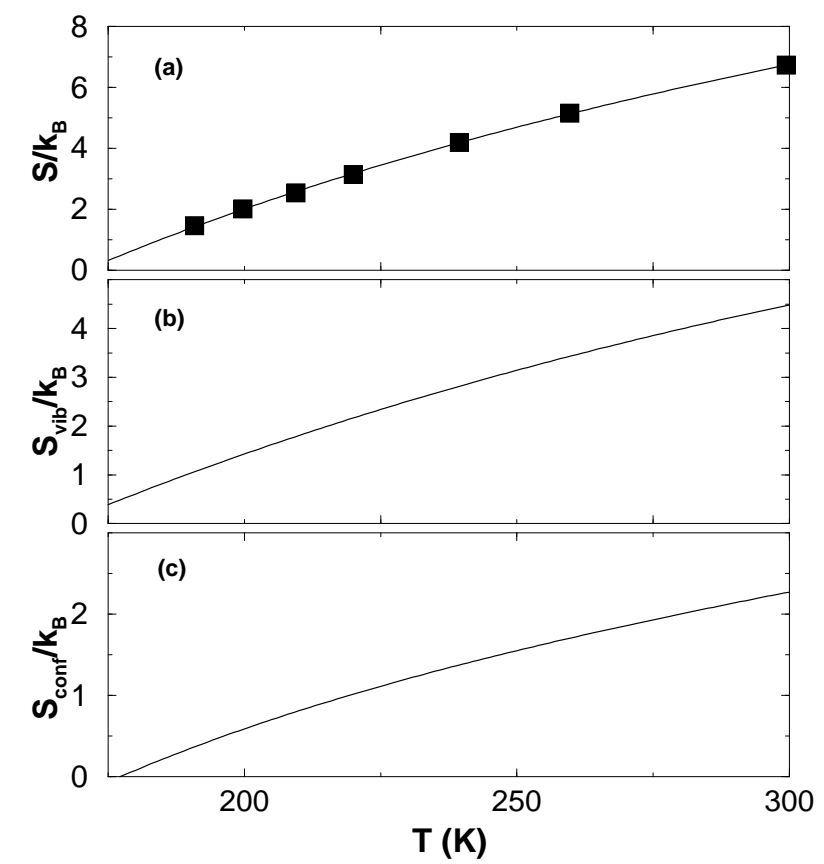

FIG. 2. Calculated values for the $\rho=1.0 \mathrm{~g} / \mathrm{cm}^{3}$ isochore of: (a) $S$ determined from simulation (filled $\square$ ). The line is a

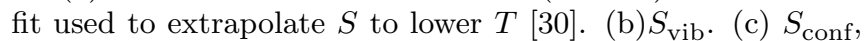
obtained by methods described in the text.

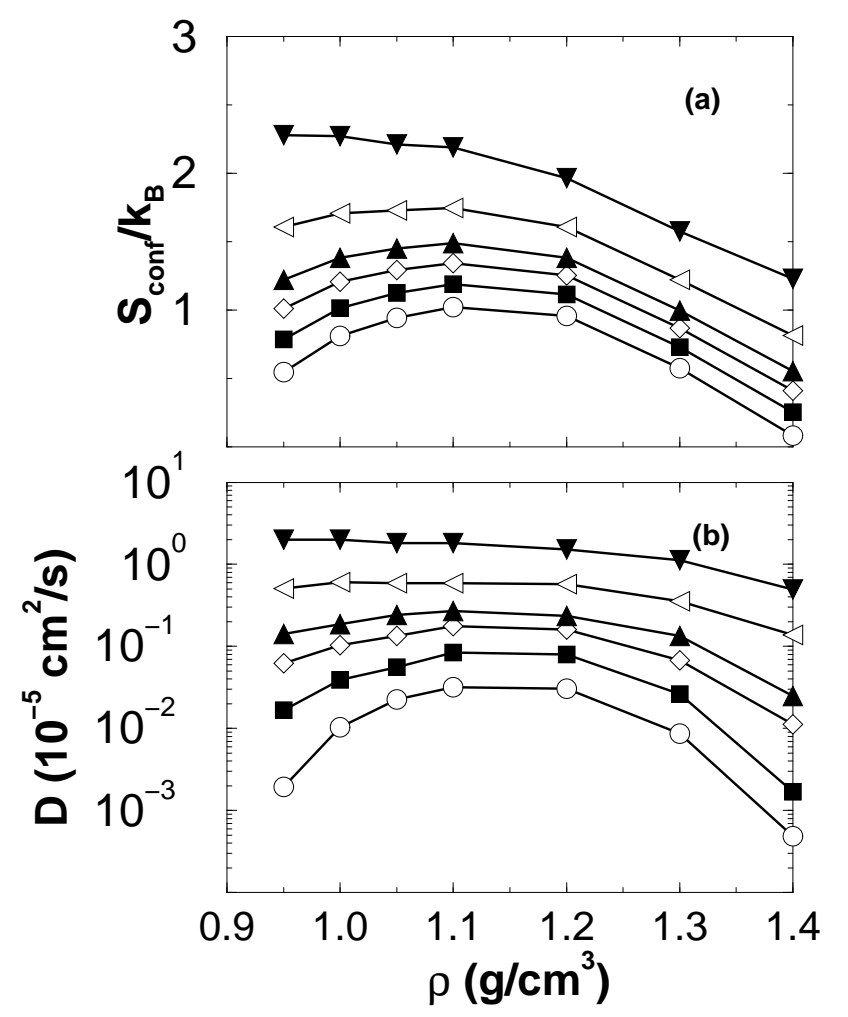

FIG. 3. (a) The configurational entropy $S_{\text {conf }}$ along isothermal paths; from top to bottom $T=300, T=260$, $T=240, T=230, T=220, T=210 \mathrm{~K}$. (b) Diffusion constant $D$ along the same isotherms. Notice the close correspondence of the maxima of $S_{\text {conf }}$ and $D$.

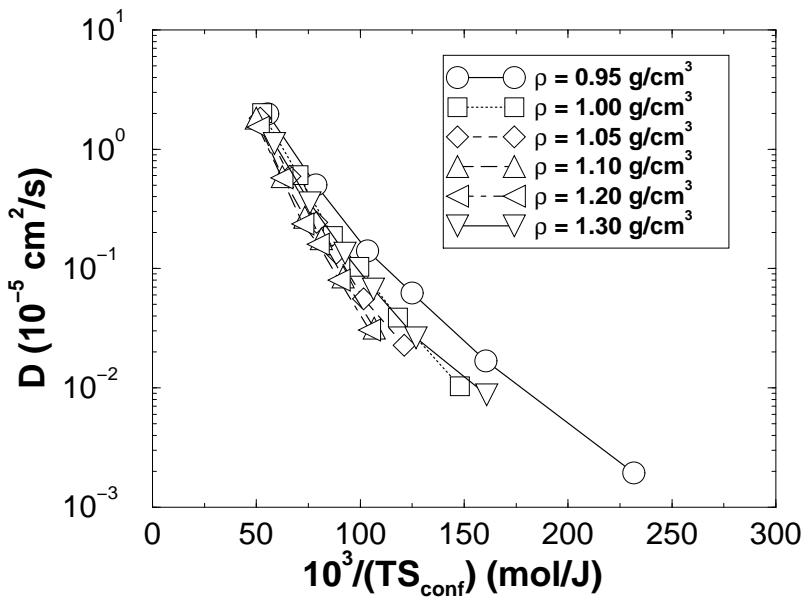

FIG. 4. Diffusion constant $D$ versus $\left(T S_{\text {conf }}\right)^{-1}$ for all studied isochores. 


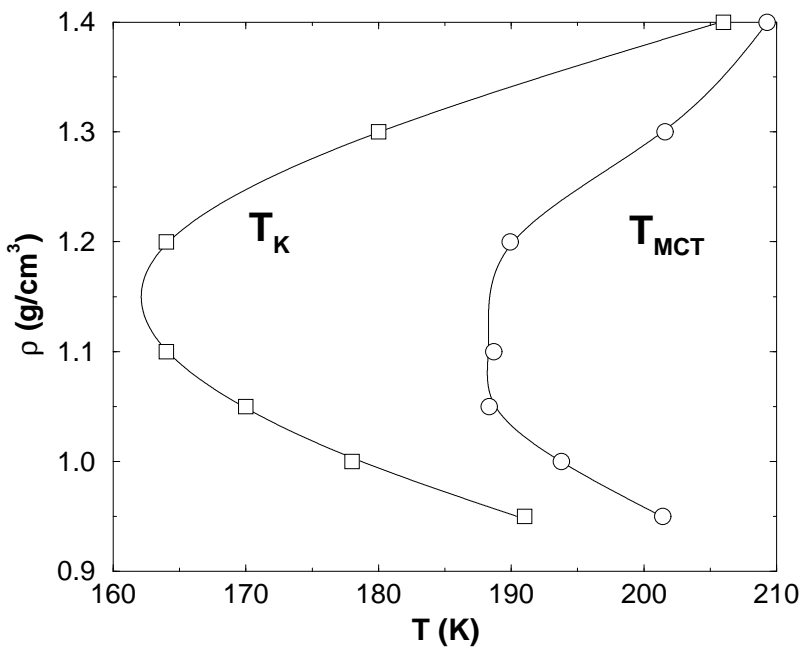

FIG. 5. The locus of the mode-coupling theory transition temperature $T_{M C T}$ (from Ref. [5]), and the locus of the Kauzmann temperature $T_{K}$. 